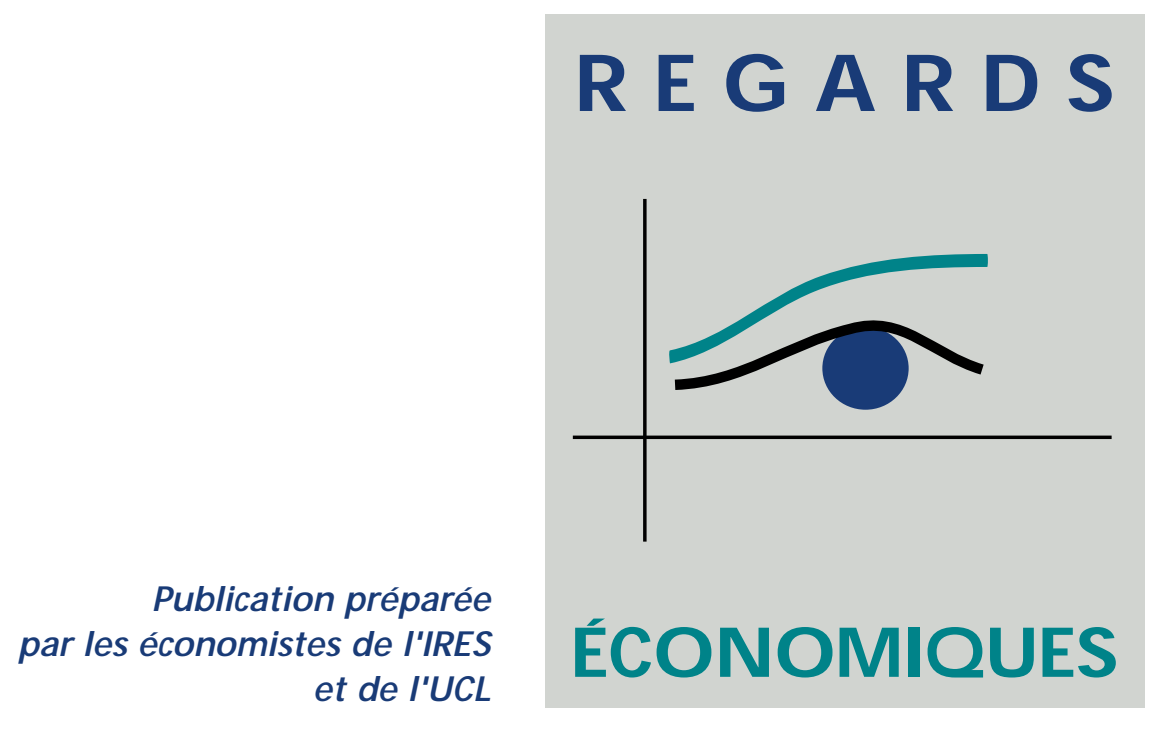

\title{
Allégements de charges sociales : une mesure à promouvoir mais à réformer. ${ }^{1}$
}

Pour répondre aux difficultés structurelles du marché $d u$ travail, y a-t-il lieu d'alléger le coût du travail par des réductions de charges sociales? Si oui, comment et jusqu'où?
Depuis plus de 25 ans, la Belgique connaît un chômage persistant, avec de fortes différences cependant selon les sous-régions et selon les catégories socio-professionnelles. Selon l'Enquête européenne sur les forces de travail de l'année 2002, le taux d'emploi parmi les travailleurs de 25 à 64 ans titulaires au plus d'un diplôme du secondaire inférieur s'élève à 48,2\% en Belgique, contre 55,2\% en moyenne chez nos voisins (Pays-Bas, Allemagne et France) ${ }^{2}$. Pour les niveaux d'études plus élevés, on n'observe guère d'écart de performance.

Pour stimuler l'emploi de travailleurs moins qualifiés, la Belgique a progressivement mis en place une panoplie de mesures incitatrices. Un allégement forfaitaire des cotisations sociales patronales existait pour les ouvriers déjà à partir de 1981. Des allégements de cotisations patronales sur les bas salaires sont introduits à partir de 1993. S'y ajoutent depuis 2000 des réductions de cotisations personnelles pour les travailleurs à bas salaires. Cet arsenal est progressivement complété par des mesures fiscales. A ces dispositifs s'ajoutent, depuis longtemps déjà, divers modes de subvention en cas d'embauches de travailleurs appartenant à divers groupes-cibles.

Le lecteur ne trouvera pas ici un inventaire détaillé des mesures existantes ${ }^{3}$ mais plutôt une réflexion sur la rationalité et les effets des principaux dispositifs évoqués à l'instant. L'accent est mis sur les allégements de cotisations sociales mais

\footnotetext{
${ }^{1}$ Nous remercions Jean Ries pour son assistance à la rédaction de ce numéro. Nous remercions également Kris Degroote (collaborateur scientifique au secrétariat du Conseil Central de l'Economie), Pierre Dmitrevsky (ONSS), Luc Masure (Bureau fédéral du Plan) et Christian Valenduc (Ministère des Finances) pour leur disponibilité. Le texte ci-dessous n'engage en rien ces personnes mais seulement ses auteurs. Nous remercions Vincent Bodart et Vincent Vandenberghe pour leurs commentaires sur une première version de ce texte.

${ }^{2}$ Calculs : Conseil Central de l'Economie.

${ }^{3}$ Le lecteur intéressé consultera http://www.meta.fgov.be/pa/paa/framesetfrbb01.htm
} 
nous évoquons aussi d'autres dispositifs qui sont, potentiellement au moins, des compléments ou des substituts à ces allégements. Cette réflexion vise à éclairer les débats et les choix des partenaires sociaux et des autorités publiques.

Pour nous, le critère de réussite ultime est le "bien-être collectif", ce qui signifie que l'emploi n'est pas une fin en soi et qu'il faut tenir compte d'éventuels objectifs en termes de juste répartition des revenus.

Nous commençons par un bref état des lieux des mesures prises en matières d'allégement du coût du travail. Puis, nous résumons notre diagnostic actuel du sousemploi belge avec une attention particulière à la problématique des moins qualifiés. Ensuite, nous abordons les résultats d'évaluations belge et internationale. Nous concluons par un ensemble de recommandations.

\section{Etat des lieux}

Les mesures d'allégement du coût du travail sont fréquemment modifiées. Le tableau 1 résume de façon sommaire l'importance et l'évolution des diverses mesures d'allégement du coût du travail.

Tableau 1 : Mesures de réduction des cotisations sociales (millions d'euros)

\begin{tabular}{lrrrrrr}
\hline & 1995 & 1999 & 2000 & 2001 & 2002 & 2003 \\
\hline Inconditionnelles (A) & 692 & 1237 & 2266 & 2667 & 2718 & 2798 \\
\hline Maribel & 449 & & & & & \\
\hline Bas salaires & 243 & & & & & \\
\hline Mesure structurelle & & 1237 & 2266 & 2667 & 2718 & 2798 \\
\hline Conditionnelles (B) & 349 & 464 & 598 & 717 & 867 & 977 \\
\hline Plans emplois* & 225 & 213 & 221 & 225 & 227 & 273 \\
\hline Redistribution du travail & & 37 & 42 & 35 & 101 & 102 \\
\hline Maribel social & & 179 & 280 & 395 & 469 & 475 \\
\hline Activation alloc. de chômage ** & & 35 & 55 & 62 & 70 & 127 \\
\hline Autres *** & 124 & & & & & \\
\hline Réductions personnelles (C) & & & 81 & 99 & 91 & 144 \\
\hline Total (A) + (B) +(C) & 1041 & 1701 & 2945 & 3483 & 3676 & 3919 \\
\hline
\end{tabular}

* Plans plus 1, plus 2, plus 3, Plan d'embauche des chômeurs, Plan d'embauche des jeunes, Plan avantage à l'embauche, Premiers Emplois, Emplois-services, Plan activa ** En 1995, cette mesure est reprise dans la rubrique "autres"

*** Plan d'entreprises et accords pour l'emploi, stage des jeunes, convention emploi-formation, engagement d'employés de maison, activation des allocations de chômage, modération salariale universités, prépensions mi-temps, interruption de carrière, semaine 38 heures, enseignement scientifique

Sources : Perspectives économiques (Bureau Fédéral du Plan), Focus Social FEB (2000), O.N.S.S. - Informations statistiques, brochure rose, 2002

Sous l'intitulé "inconditionnelles", on retrouve les mesures de réduction des charges patronales sans condition d'embauche (le stock des travailleurs occupés est donc aussi concerné) ni limitation de durée. Les mesures “conditionnelles" sont, avec des nuances, fonction du nombre de recrutements et limitées dans le temps (la subvention disparaît après une certaine période d'occupation du travailleur). Les "réductions personnelles" sont des allégements de cotisations sociales personnelles accordées aux travailleurs à bas salaire. Les allégements "inconditionnels" représentent la plus grande part des dépenses. Leur importance relative est 


\section{... Etat des lieux}

Figure 1 : Evolution de la réduction de cotisations sociales patronales en fonction du revenu mensuel brut (ouvriers) maximale en 2000 (77\% du montant total des réductions). Par la suite, la croissance plus forte des dépenses "conditionnelles" et l'introduction des allégements de cotisations personnelles réduisent la part des dépenses inconditionnelles (71\% en 2003). Les allégements "inconditionnels" ont, dans le passé, été réservés aux ouvriers (programme Maribel) et aux travailleurs à bas salaires. Depuis 1999, ces deux dispositifs ont fusionné pour devenir les "mesures structurelles". Cette fusion a introduit une séquence d'ajustements réglementaires.

La figure 1 illustre la façon dont sont calculées les réductions inconditionnelles. La figure est basée sur la législation en vigueur au second trimestre de l'année 2002 et concerne les ouvriers (qui représentent les trois-quarts des travailleurs "à bas salaire"). Le profil est similaire pour les employés. Schématiquement, le lien entre le salaire mensuel brut et la réduction des cotisations patronales a la forme d'un $\mathrm{U}$ inversé. Tous les travailleurs bénéficient d'une réduction de cotisations patronales forfaitaires de $127 € /$ mois ( $105 € /$ mois pour les employés). Pour les salaires compris entre $855 € /$ mois et $1.735 € /$ mois $(855 €$ et $1.583 €$ respectivement pour les employés), l'allégement est majoré et atteint un maximum de $245 € /$ mois ; il revient ensuite progressivement à la valeur forfaitaire applicable à tous.

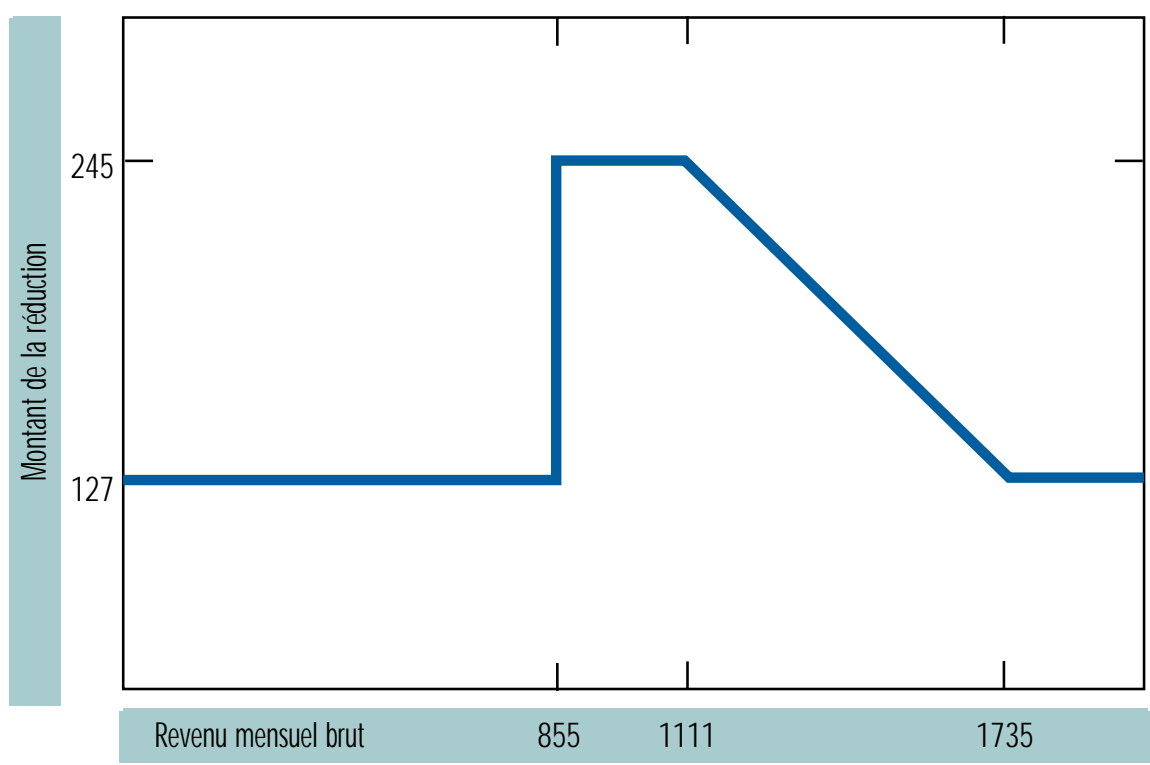

Soulignons qu'un revenu mensuel brut de $855 €$ correspond à $75 \%$ du revenu mensuel minimum moyen garanti de base et est également (après déduction de quelque $13 \%$ de cotisations personnelles et des impôts des personnes physiques) sensiblement inférieur à la valeur des indemnités de chômage perçues par un chef de ménage ( $827 € /$ mois au 1er janvier 2002). La proportion de travailleurs avec revenu mensuel brut inférieur à $855 €$ est donc faible. En revanche, la proportion de travailleurs ayant un revenu brut supérieur à $1.735 € /$ mois $(1.538 € /$ mois pour les employés) est élevée (quelque 73\%). Seulement 520.000 travailleurs (soit $11 \%$ de la population active) bénéficient d'une réduction de cotisations patronales majorée. Le maintien d'une réduction forfaitaire de $127 € /$ mois (105€ pour les employés) au-delà du seuil de $1.735 € /$ mois ( $1.538 € /$ mois pour les employés) sans aucune limite de revenu implique que la toute grande majorité des dépenses de réductions inconditionnelles $(69 \%)$ est consacrée à des revenus relativement élevés. Seuls $9 \%$ du budget total sont alloués aux majorations de réduction spécifiques aux bas salaires. ${ }^{4}$

\footnotetext{
${ }^{4}$ Ces chiffres sont approximatifs (les statistiques de l'ONSS ne permettent pas un calcul précis) mais néanmoins révélateurs.
} 
... Etat des lieux

\section{Diagnostic}

\section{L'importance des "institutions" du marché du travail}

\section{Coin fiscal, cô̂t du travail
et chômage \\ Coin fiscal, coût du travail
et chômage}

De 1970 à 1996, le “coin fiscal”, c'est-à-dire l'écart moyen entre salaire-coût et salaire-net dû à la fiscalité (impôt des personnes physiques) et à la parafiscalité (cotisations à la sécurité sociale), n'a cessé de croître dans les pays de l'Union européenne. En Belgique, l'écart est passé de $34 \%$ à 52\% du salaire-coût. Il a depuis (très) légèrement diminué. Jusqu'en 1982, cette hausse du coin fiscal est due pour l'essentiel à la hausse de l'impôt sur les personnes physiques ; après 1982, elle est due pour l'essentiel au relèvement des taux de cotisations patronales. Une telle évolution peut-elle avoir contribué significativement à la hausse du chômage observée sur cette période ?

On admet généralement qu'à long terme, l'impact économique ne dépend pas du côté du marché qui formellement paie les contributions et les impôts. Une variation des cotisations personnelles à la sécurité sociale et une variation de cotisations patronales de même ampleur doivent donc, à terme, avoir les mêmes effets sur le salaire net, le coût salarial et l'emploi ("neutralité de l'incidence fiscale").

L'effet sur l'emploi d'une hausse du coin fiscal peut être nul ou élevé, en fonc- 
... Diagnostic

Chômage de longue durée : cause ou conséquence de la persistance du chômage tion de son impact sur les exigences salariales des travailleurs. L'impact sur le coût salarial et l'emploi sera nul lorsque le prélèvement est perçu comme un revenu différé (pensions de retraite, assurances, etc.) plutôt qu'un impôt. L'impact sera négligeable également lorsque la hausse des prélèvements affecte les revenus des travailleurs en emploi et celui des travailleurs en chômage de la même façon, parce que la baisse des revenus nets du chômeur exerce un impact négatif sur les exigences salariales. Dans les autres cas, la hausse de la (para-)fiscalité est susceptible d'engendrer des pressions salariales accrues, les salariés cherchant à récupérer par une hausse du salaire-coût la perte de pouvoir d'achat engendrée par la taxation. A court terme, il en résulterait à la fois une hausse du salaire-coût et une baisse du salaire net. Lorsque les marchés sont parfaitement concurrentiels, le partage entre ces deux effets est fonction des élasticités respectives de l'offre et de la demande de travail. Dans les autres cas de figure, plus réalistes, les pouvoirs de négociation et les caractéristiques institutionnelles, telles que le salaire minimum, doivent être pris en compte. La baisse de l'emploi sera d'autant plus forte que la hausse de la taxation est reportée à court terme sur le salaire-coût et que la demande de travail est sensible au coût du travail. A long terme, l'effet négatif sur l'emploi est accentué par la baisse de la profitabilité et de l'investissement.

Plusieurs travaux empiriques suggèrent que la hausse du coin fiscal a contribué positivement mais faiblement à la hausse observée des taux de chômage au cours des trente dernières années (en particulier Blanchard-Wolfers, 2000) ; d'autres suggèrent des effets nettement plus importants. Ces résultats empiriques contrastés traduisent la difficulté d'appréhender correctement les comportements salariaux et les différences entre court et long terme.

Comparés aux Etats-Unis, la plupart des pays européens sont caractérisés par une forte proportion de chômeurs de longue durée. En Belgique, quelque 40 \% des chômeurs enregistrés sont inoccupés depuis plus de deux ans.

Lorsque l'on suit l'évolution de travailleurs entrés en chômage au même moment, on observe que le taux de sortie du chômage s'amenuise au fil du temps. Tout semble se passer comme si le chômeur qui n'a pas eu la chance de trouver un emploi rapidement voyait son employabilité réduite de mois en mois. La perte d'employabilité pourrait refléter une réelle perte de savoir-faire et de capacité d'insertion, à mesure que la durée d'inoccupation se prolonge. Elle pourrait également être la conséquence du seul comportement d'embauche des entreprises, lorsque celles-ci interprètent systématiquement le chômage de longue durée comme un signal négatif à propos du travailleur. Dans un tel scénario, un pays qui aurait vu s'élever progressivement la proportion de chômeurs de longue durée resterait bloqué dans une situation de chômage élevé, les travailleurs ayant progressivement perdu leur employabilité se retrouvant de facto exclus du marché du travail. Le chômage de longue durée serait en lui-même cause de la persistance du chômage.

Cette hypothèse de "dépendance à la durée" a été testée, d'abord sur données macroéconomiques, ensuite sur données microéconomiques, au fur et à mesure que des informations sur les trajectoires et les caractéristiques individuelles des chômeurs devenaient disponibles. Ces tests économétriques sur données individuelles conduisent généralement à minimiser le rôle de la dépendance négative à la durée. La baisse de la probabilité de sortie avec la durée de chômage est apparente et reflète un processus de tri : les travailleurs à caractéristiques favorables quittent le chômage en premier. Ce n'est pas la durée du chômage qui réduit la probabilité de sortie : deviennent chômeurs de longue durée ceux qui, dès leur 
... Diagnostic

Chômage des peu qualifiés : changement de la demande ou effet d'éviction entrée en chômage, ont des caractéristiques (niveau de qualification, localisation géographique, par exemple) qui nuisent à la (ré)insertion en emploi (Machin et Manning, 1999, Cockx et Dejemeppe, 2002, Dejemeppe, 2003). En d'autres termes, le chômage de longue durée est une conséquence plutôt qu'une cause de la persistance de taux de chômage élevés.

Le chômage ne touche pas toutes les catégories de travailleurs de la même façon. Si les taux de chômage ont augmenté pour presque toutes les catégories de travailleurs, il a augmenté beaucoup plus pour les travailleurs moins qualifiés (en termes de niveau d'études). Le taux de chômage des travailleurs avec un diplôme du secondaire inférieur est ainsi passé de $5 \%$ à $14 \%$ entre 1977 et 1996 (de 1,3\% à $2,5 \%$ pour les universitaires).

\section{- Baisse de la demande relative de main-d'ouvre moins qualifiée}

Cette évolution contrastée des taux de chômage peut résulter d'un changement dans la demande relative de main-d'œuvre qualifiée, suite par exemple à l'introduction de technologies nouvelles, aux changements organisationnels (autonomie accrue, adaptabilité à différentes tâches, etc.) ${ }^{5}$, aux effets de mondialisation (concurrence des pays à bas salaire et les changements de spécialisation à l'échelle mondiale). Dans le même temps, l'offre de qualifications a bien sûr ellemême fortement augmenté. Plusieurs études empiriques suggèrent cependant un accroissement significativement plus important de la demande, et donc un biais net défavorable aux moins qualifiés. Sur des marchés du travail parfaitement concurrentiels, la conséquence serait une baisse du salaire relatif des moins qualifiés. L'existence de salaires minima et/ou de garanties de ressources (indemnités de chômage ou autres formes de transferts dans les pays où le droit aux indemnités de chômage est limité dans le temps) réduit cependant la flexibilité des salaires relatifs, avec comme conséquence un chômage plus important pour les moins qualifiés.

Reste à vérifier quelle est la contribution de ce problème à la hausse observée du chômage au cours des trente dernières années. Nombre d'auteurs suggèrent un effet significatif mais relativement peu important, et soulignent par ailleurs que si les effets asymétriques de la croissance étaient la cause majeure du chômage, on aurait dû observer, en même temps qu'une hausse du chômage des moins qualifiés, une baisse de celui des plus qualifiés, ce qui n'est pas le cas si l'on regarde la tendance au cours des trente dernières années. Ce raisonnement ne tient pas suffisamment compte, cependant, des interactions entre les deux segments du marché du travail, notamment via les négociations salariales et les rigidités observées de salaires relatifs. Si le type de croissance et de progrès technique observé implique des gains de productivité plus importants pour les plus qualifiés et pousse les salaires de ces derniers à la hausse, les salaires relatifs ne pourront demeurer inchangés que si les demandes salariales des moins qualifiés sont elles aussi révisées à la hausse. Cette pression salariale accrue détériore la profitabilité des entreprises et exerce un effet défavorable sur la demande des deux types de main-d'œuvre, susceptible d'engendrer une hausse généralisée des taux de chômage.

\section{- Baisse de la demande globale de main-d'ouvre et effet d'éviction}

Nombre de travaux suggèrent par ailleurs qu'une proportion significative d'emplois exigeant a priori une faible qualification est occupée par des travailleurs surqualifiés. La mesure du phénomène est très délicate, mais des pourcentages allant

${ }^{5}$ Voir par exemple Bresnahan et al. (2002) et Caroli et van Reenen (2001). 


\section{... Diagnostic}

Comportements de recherche d'emploi et "trappes à chômage"

\section{Evaluations}

de $10 \%$ à $30 \%$ sont considérés comme plausibles. Ce constat suggère que la forte hausse des taux de chômage des moins qualifiés pourrait être la conséquence d'un effet d'éviction plutôt que d'un changement de la demande défavorable aux moins qualifiés. Lorsque la situation sur le marché du travail se détériore, les chômeurs les plus qualifiés ont l'avantage de pouvoir postuler à la fois pour des emplois plus qualifiés et pour des emplois moins qualifiés. Dans ce scénario, la hausse plus forte du taux de chômage des moins qualifiés est la simple conséquence de la baisse généralisée de la demande de travail.

Les deux scénarios, changement de la demande relative et effet d'éviction, peuvent bien sûr coexister. Utilisant un modèle macroéconomique calibré sur données belges, Pierrard et Sneessens (2003a) montrent qu'on peut expliquer la quasi totalité de la hausse observée des taux de chômage des travailleurs plus et moins qualifiés par les changements observés dans la demande et l'offre relative de main-d'œuvre qualifiée. La rigidité des salaires relatifs joue un rôle crucial ; elle est la cause première de la hausse des taux de chômage. Les effets d'éviction jouent cependant, eux aussi, un rôle important, en accentuant les effets de la rigidité des salaires relatifs. Sans ces effets d'éviction, la hausse des taux de chômage serait réduite de moitié.

Le taux de chômage dépend également des comportements de recherche d'emploi des personnes sans-emploi et de leurs réponses aux offres éventuelles. L'intensité de la recherche d'emploi est influencée négativement par le niveau des allocations et par le coût de la recherche, et elle est influencée positivement par le rythme d'arrivée des offres et par le gain escompté en cas de reprise d'emploi. Les études étrangères disponibles concluent que la probabilité d'acceptation d'une offre d'emploi est très élevée parmi les chômeurs et que la rapidité du retour vers l'emploi dépend d'abord de la fréquence d'arrivée des offres d'emploi. Les études sur données belges montrent cependant qu'en Belgique l'impact de l'assurance-chômage sur le comportement de recherche et sur le rythme de sortie du chômage n'est pas à minimiser ${ }^{6}$. En revanche, le phénomène de "trappe à chômage" induit par un niveau de salaire offert trop faible par rapport à l'indemnité de chômage ne semble important que pour une minorité de travailleurs (voir d'Addio et al, 2002).

Dans la section précédente, nous avons résumé notre compréhension des causes du chômage structurel. Sans avoir pu aborder les spécificités régionales, nous avons conclu à l'importance de plusieurs mécanismes : les chocs de demande de travail défavorables aux peu qualifiés, les phénomènes d'éviction, les mécanismes de formation des salaires et les incitations à la recherche d'emploi. Nous en venons à présent aux évaluations des politiques. Nous distinguerons trois groupes : les dispositifs visant à réduire de manière permanente le coût du travail, ceux qui subventionnent de manière temporaire le recrutement et enfin ceux qui visent à combattre les "trappes à chômage". La neutralité de l'incidence fiscale évoquée ci-dessus permet de conclure que ces distinctions ont un caractère artificiel. Elles correspondent cependant à des intentions distinctes dans le chef des décideurs économiques.

\footnotetext{
${ }^{6}$ Voir Dor et al (1997), d'Addio et al (2002), Van der Linden et Dor (2002), Van der Linden (2003), Cockx et Ries (2003). Comme un nombre croissant d'études étrangères, ces derniers obtiennent que la fin de droit aux indemnités de chômage stimule sensiblement la réinsertion en emploi (en l'occurrence, de femmes chômeuses de longue durée). Une baisse du niveau des indemnités de chômage et de la durée de droit à ces allocations pourrait ainsi, indépendamment de la cause, contribuer à résorber le chômage.
} 
Les dispositifs visant à réduire de manière permanente le coût du travail
La sensibilité (appelée encore l'élasticité) de l'emploi au coût du travail fait l'objet d'études depuis de nombreuses années. Elle varie fort selon que l'on se situe au niveau de la firme, du secteur ou de l'économie entière. Elle varie aussi fortement selon que l'on regarde le travailleur moyen ou des sous-groupes. Lorsqu'on prend en compte les effets du coût du travail sur le volume de l'activité économique, une élasticité de l'emploi au coût du travail proche de -1 apparaît raisonnable pour les moins qualifiés (Cahuc, 2002). Toute chose égale par ailleurs (progrès technique, taux d'intérêt, prix des inputs intermédiaires, etc.), une hausse d' $1 \%$ du coût du travail réduit donc à terme leur emploi d'environ $1 \%$. L'élasticité est en général moins forte pour les travailleurs plus qualifiés, pour lesquels il est plus difficile de trouver des substituts dans le processus de production.

On a indiqué ci-dessus qu'une mesure (para-)fiscale qui s'applique à un groupe précis d'individus (employeurs, travailleurs) peut effectivement être à charge de ce groupe comme elle peut être (partiellement ou totalement) reportée sur l'autre côté du marché. Van Poeck et al (1996), Ooghe et al (2000) et Joyeux et Stockman (2002) aboutissent à la conclusion que les hausses de cotisations patronales sont dans une large mesure compensées par une diminution des salaires nets (voir également Burggraeve et Du Caju, 2003). Ces études économétriques impliquent que l'emploi répond faiblement à une variation proportionnelle du "coin fiscal" ou des taux qui le composent. En agissant surtout sur le niveau des salaires, les allégements de cotisations patronales des années nonante auraient donc surtout stimulé la croissance des salaires nets et dès lors incité les taux de participation au marché du travail à se redresser. Ces conclusions sont cependant hâtives. Les mesures mises en place dans les années nonante n'introduisent en effet pas un changement proportionnel. (voir le profil de la figure 1). Or, à niveau donné du coin fiscal, une réforme qui augmente la progressivité 7 de la (para-)fiscalité aura un effet modérateur sur les salaires négociés (Cahuc et Zylberberg, 2001, p. 542-547). L'intuition est la suivante. A taux moyen donné de la (para-) fiscalité, une hausse des taux marginaux de taxation (ou de cotisation sociale) a pour conséquence qu'une hausse (marginale) du salaire brut "rapporte moins à l'employé qu'elle ne coûte à l'entreprise. Cette dernière voit son profit plus affecté que par le passé par les hausses de salaire, ce qui, au cours des négociations, contribue à freiner les revendications salariales" (Cahuc et Zylberberg, 2001, p. 544). Les effets des réformes des années nonante sur la progressivité du système (para-)fiscal n'ont pu être étudié par les auteurs mentionnés ci-dessus.

Venons-en à présent aux résultats d'évaluation des allégements de cotisations patronales. Appliquant une méthode quasi-expérimentale (Heckman et al., 1999), Crépon et Desplatz (2001) ont étudié l'impact d'une extension du dispositif français d'allégement des cotisations patronales en 1995 et 1996. En pourcentage du salaire brut, les allégements ont été augmentés substantiellement de $6 \%$ à $18 \%$ au niveau du salaire minimum (le "SMIC"). Cette exonération est dégressive en fonction du salaire et devient nulle au niveau de 1,3 fois le SMIC. Selon l'étude, entre 255.000 et 670.000 emplois (= entre 1 et $2,5 \%$ de la population active), non qualifiés et, dans une moindre mesure, qualifiés, auraient été créés ou sauvegardés à la fin $1997 .{ }^{8}$

Les simulations de modèles sont nombreuses en Belgique. Aussi, nous limite-

\footnotetext{
${ }^{7}$ Un allégement forfaitaire est progressif car le montant du forfait diminue en proportion du revenu. Ainsi, l'élasticité du coût salarial par rapport au salaire brut est supérieure à l'unité. Cette même propriété s'observe dans la zone où l'allégement majoré décroît. ${ }^{8}$ Cette étude a suscité de nombreuses réactions soulignant les limites de la méthode adoptée. Ces critiques ont toutefois été parées (voir Cahuc (2002), p. 14-15, pour une discussion).
} 


\section{... Evaluations}

rons-nous à trois études postérieures à celle de Van Poeck et al (1998). ${ }^{9}$ Les résultats de simulation de modèles diffèrent par ce que les hypothèses de modélisation et les méthodes d'analyse ne sont pas les mêmes. De plus, l'ampleur des allégements simulés diffère. Aussi, pour faciliter la lecture, avons-nous résumé les effets de trois études récentes en extrapolant de manière purement linéaire les résultats obtenus. Plus précisément, on a ramené les effets au cas d'un allégement ex ante (sur les travailleurs à "bas salaire") équivalent à $1 \%$ du PIB. ${ }^{10}$ Une annexe fournit plus de précision sur chaque étude. Il s'agit chaque fois d'effets à moyen ou long terme sur l'emploi (en nombre d'unités).

Tableau 2 : Impact extrapolé sur l'emploi d'un allégement des cotisations patronales sur les travailleurs à bas salaires équivalent ex ante à $1 \%$ du PIB.

\begin{tabular}{llll}
\hline Etude & $\begin{array}{l}\text { Emploi } \\
\text { peu qualifié }\end{array}$ & $\begin{array}{l}\text { Emploi } \\
\text { qualifié }\end{array}$ & Effet total \\
\hline Sneessens et Shadman-Mehta (2000) * & 83.000 & 33.000 & 116.000 \\
\hline Stockman (2002) * & & $\begin{array}{l}47.600 \text { (salaires bruts } \\
\text { librement négociés) } \\
80.800 \text { (plein effet des allége- } \\
\text { ments sur le coût du travail) }\end{array}$ \\
\hline Pierrard et Sneessens (2003b) ** & 45.300 & 12.700 & $58.000 * * *$ \\
\hline
\end{tabular}

* Simulation sans prise en compte d'un financement compensatoire visant à stabiliser les recettes de la Sécurité Sociale.

** Simulation avec prise en compte d'un financement compensatoire visant à stabiliser les recettes de la Sécurité Sociale.

*** L'effet est augmenté de $20 \%$ si le financement compensatoire s'opère par une hausse de la (para)-fiscalité sur les salaires des travailleurs qualifiés.

Même si l'évidence empirique ne soutient pas que la persistance du chômage soit essentiellement causée par un enlisement dans le chômage de longue durée (cf. la section précédente) ${ }^{11}$, une subvention temporaire, qui s'annule après une certaine période d'occupation du travailleur, pourrait être plus efficace qu'un allégement permanent des cotisations sociales ciblé aux travailleurs faiblement qualifiés. En effet, si le travailleur augmente sa productivité par un apprentissage sur le tas, la subvention devient progressivement une perte sèche : le travailleur maintient son emploi même en l'absence de la subvention (Orszag et Snower, 2003). Toutefois, il est maintenant bien établi que la progression des salaires, et donc - avec des nuances - celle de la productivité, est lente parmi les travailleurs peu qualifiés (Blundell et Meghir, 2002, p.23). Ceci est compatible avec les résultats récents d'évaluation. Ceux-ci indiquent que le taux de licenciement augmente sensiblement après la fin de la période où l'occupation est subventionnée ${ }^{12}$ et que la durée des périodes d'emploi ultérieures n'est pas affectée

\footnotetext{
${ }^{9}$ Voir Van Poeck et al (1998) pour une synthèse de la littérature plus ancienne. On consultera également Hendrickx et al (2003), rapport à la base de plusieurs communications lors du colloque "Evaluations macro et micro-économiques de politiques d'emploi" organisé par le Bureau fédéral du Plan le 26 septembre 2003.

${ }^{10}$ Cette approche est assez sommaire car les effets ne sont pas linéaires. De surcroît, vu la croissance du PIB, le niveau total de l'allégement dépend de l'année où la mesure entre en vigueur (1\% du PIB représente en 2001 environ 2,5 milliards $€$ ). La notion de "peu qualifié" ou de "bas salaire" n'est en outre pas identique dans chaque simulation. Néanmoins, le tableau 2 devrait fournir des ordres de grandeur.

${ }^{11}$ Un emploi temporaire préviendrait l'enlisement et ainsi la persistance du chômage.

${ }^{12}$ Les résultats préliminaires de l'impact du Plan d'Embauche sur des jeunes chômeurs de longue durée en Belgique vont dans le même sens (Cockx et al., 2004).
} 


\section{... Evaluations}

\section{Dispositifs luttant contre les trappes à chômage}

(Bolvig et al., 2003). Une subvention temporaire, ciblée sur les travailleurs faiblement qualifiés, risque donc de ne pas stimuler l'emploi durablement.

D'autres auteurs ont néanmoins conclu que des subventions temporaires aboutissent à une augmentation du taux de réinsertion dans un emploi non subventionné pour autant que les subventions temporaires soient réservées à des emplois du circuit économique "normal" (Martin et Grubb, 2001 ; Calmfors et al., 2002 ; Gerfin et al., 2002 ; Lalive et al., 2002) et pour autant que la subvention ne dure pas. Une subvention temporaire semble donc efficace parce qu'elle réduit les coûts de sélection des candidats et non pas parce qu'elle permet de renforcer progressivement la productivité du travailleur. Par conséquent, une subvention temporaire ne se justifierait que pour des courtes périodes, disons de maximum 6 mois.

Plus que d'autres politiques d'activation, les subventions sont critiquées pour leurs effets indirects. En ciblant la subvention sur des groupes de travailleurs spécifiques (les jeunes, les travailleurs âgés, les chômeurs de longue durée,...), l'impact favorable pour ces groupes risque de se faire aux dépens de demandeurs d'emploi n'ayant pas bénéficié de la politique d'activation ("effet de substitution") ou de travailleurs licenciés à cause de la compétition induite (directement ou indirectement) par le travailleur bénéficiant de l'activation (“effet de déplacement"). A l'inverse, en absence de ciblage, comme dans le cas d'une baisse généralisée des cotisations sociales, le travailleur serait souvent embauché même en absence de l'aide ("d'effet d'aubaine" ou "d'effet de perte sèche"). ${ }^{13}$ La littérature trouve que ces effets indirects sont généralement importants (Martin et Grubb, 2001 ; Calmfors et al., 2002 ; pour la Belgique, voir Van der Linden, 1997). Lorsque le bénéficiaire de la subvention connaît un risque de chômage élevé et lorsque la population des non-bénéficiaires n'a pas un niveau de productivité similaire, les risques de perte sèche et d'effet de substitution ou de déplacement deviennent cependant fort faibles. Une subvention ciblée sur les bas salaires rencontre bien cette exigence.

Si la trappe à chômage était la cause principale du chômage structurel des peu qualifiés, on pourrait argumenter qu'une stimulation de l'offre de travail de ce segment de travailleurs aurait plus d'impact qu'un renforcement de la demande. Une telle stimulation s'effectuerait par exemple via le "bonus crédit d'emploi" proposé dans le récent accord gouvernemental. Des études récentes ${ }^{14}$ sur l'effet des crédits d'impôts au profit des personnes à faible revenu aux Etats-Unis ("Earned Income Tax Credit" ou "EITC") et au Royaume-Uni ("Working Families Tax Credit") aboutissent toutefois à des conclusions mitigées. D'une part, ces dispositifs ont effectivement permis d'augmenter le taux d'activité de personnes célibataires et des couples dont les deux membres sont inactifs. A l'inverse, ils ont diminué la participation des personnes dont le conjoint travaille et diminué la durée de travail de personnes qui ainsi réduisent leur revenu afin de bénéficier de l'avantage du crédit d'impôt. Cahuc (2001) argumente que les contextes institutionnels différents des pays anglo-saxons et de l'Europe continentale ne permettent pas de transposer ces résultats. Le niveau du salaire minimum et la protection sociale étant plus généreux en Europe continentale, l'emploi d'une grande proportion des travailleurs faiblement qualifiés y est contraint par manque de demande de travail à ce niveau de salaire. Un accroissement de l'offre ne pourrait qu'exacerber le chômage de ce groupe.

On pourrait évidemment argumenter que le chômage élevé en Europe continen-

\footnotetext{
${ }^{13}$ Le ciblage peut aussi être inapproprié et favoriser l'effet d'aubaine. Le tableau 5 de Lopez-Novelle (2003) le suggère fort clairement dans le cas des Plan Plus.

${ }^{14}$ Voir Cahuc (2001) pour une synthèse.
} 


\section{... Evaluations}

\section{Conclusions}

\section{Priorité à la création d'emploi pour les moins qualifiés}

\author{
Réformer le système \\ des allégements structurels
}

tal est in fine causé par cette protection social généreuse (cfr. la dernière partie de la section 3). Bien que nous estimions que l'efficacité de l'assurance-chômage peut être améliorée par un réaménagement du profil des indemnités (cfr. Cockx et Ries, 2003), nous ne pensons pas que le chômage structurel puisse baisser de manière appréciable sans une baisse significative du niveau moyen de l'indemnité. Puisqu'une telle politique risque d'augmenter sensiblement le taux de pauvreté, elle n'est pas, selon nous, une option à prendre en considération.

Les faits, le diagnostic et les évaluations résumés ci-dessus nous conduisent aux propositions suivantes:

La conclusion de notre diagnostic est que la priorité doit être donnée à la création de postes de travail pour les personnes moins qualifiées. Dès lors, les allégements de charges patronales devraient être concentrés sur une fourchette assez étroite de bas salaires pour avoir le plus d'effet au moindre coût. De l'autre côté de la distribution des salaires, un plafonnement des cotisations sociales va fort probablement entraîner des pressions salariales sur ce segment du marché du travail et aura donc des effets négligeables sur l'emploi. Dans son volet relatif aux barèmes supérieurs d'imposition, la récente réforme fiscale devrait avoir des effets similaires.

La figure 2 schématise la réforme proposée pour un ouvrier. ${ }^{15}$ Nous proposons de réformer le dispositif d'allégement structurel de sorte qu'on élimine toute cotisation patronale sur les salaires inférieurs ou égaux au $1.111 € /$ mois. A titre de comparaison, le salaire minimum de base brut était de $1.140 € /$ mois en janvier 2002 . Au-delà de ce niveau de salaire, l'allégement devrait décroître progressivement. L'allégement forfaitaire actuel est maintenu au-delà d'un salaire mensuel brut de $1.735 €$ pour les ouvriers et $1.538 €$ pour les employés.

Afin de mieux appréhender les implications d'une telle réforme, nous avons entrepris d'en simuler, fût-ce sommairement, les effets. Confrontés à l'absence de plusieurs informations, nous avons dû baser nos calculs sur certaines hypothèses. Nous supposons que l'élasticité de la demande au coût salarial se situe entre 0,75 et - 1 dans ce segment du marché du travail. Nous ne tenons pas compte de l'effet de la réforme sur le salaire négocié ni de l'impact positif sur l'emploi de travailleurs à salaires plus élevés. Les résultats de cette simulation sont donc à considérer avec prudence. Ils n'offrent qu'un ordre de grandeur des effets.

Le coût ex ante d'une telle réforme serait de l'ordre de 415 millions $€$ par an $(0,15 \% \mathrm{du} \mathrm{PIB})$, ce qui correspond à un peu plus de la moitié du budget prévu pour les allégements supplémentaires dans le récent accord gouvernemental. ${ }^{16}$ Elle permettrait de créer ou de sauvegarder de 27.000 à 36.000 postes de travail correspondant à un nombre allant de 16.000 à 21.000 emplois en équivalent temps plein (EFT). Le coût ex ante est donc de 19.000 à $26.000 €$ par an par EFT supplémentaire. Ex post, le coût sera toutefois plus faible, puisque 1'Etat économise : bien que celui-ci doive financer les allégements des nouveaux emplois créés, les indemnités de chômage ne sont plus dues et des taxes sont perçues sur la rémunération additionnelle. Nous estimons cette économie à 7.250€ par an par EFT supplémentaire. Le coût ex post serait donc situé entre 12.000 et $18.500 €$ par EFT et entre 7.200 et $11.000 €$ par poste. Le coût net de la réforme serait ainsi de l'ordre de 260 millions $€$ par an.

\footnotetext{
${ }^{15}$ L'allure de la courbe serait la même pour les employés. Toutefois, comme le taux de cotisations patronales est nettement plus faible pour les employés, la réduction maximale est $384 €$ par mois pour les employés.

${ }^{16}$ Pour des motifs budgétaires, le gouvernement a décidé de réduire le budget de 800 à 400 millions $€$ pour l'année 2004.
} 
Figure 2 : Allégements de cotisations patronales pour les ouvriers : situation initiale (en bleu) et réforme proposée (en vert)

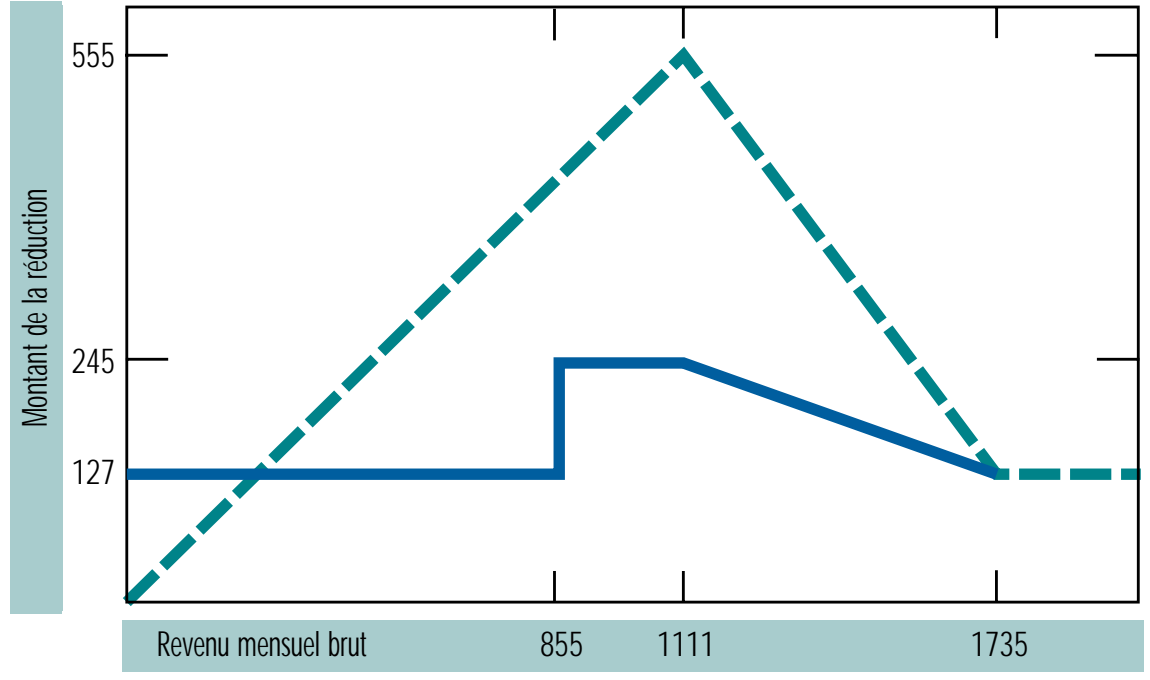

Comme indiqué ci-dessus, nous avons, pour la simplicité, maintenu l'allégement forfaitaire au-delà d'un salaire de $1.750 € /$ mois. Une alternative, que nous estimons meilleure pour l'emploi, consisterait à réduire l'allégement d'une manière moins abrupte et à l'annuler au-delà d'un seuil (disparition de l'allégement forfaitaire).

Si la réforme proposée était en outre d'application aux employeurs qui sont des personnes physiques, elle serait efficace dans la lutte contre le travail au noir à bas salaire.

Il ne faut pas en rester là. Nous proposons d'instaurer le même allégement aux travailleurs à bas salaire du secteur non-marchand et ceci, selon les mêmes modalités que pour le secteur marchand, afin d'assurer la transparence du système. En effet, s'il s'agit de promouvoir l'emploi, il n'y a pas lieu de différentier la subvention selon le secteur. Cela dit, il y a des raisons d'octroyer un financement spécifique à des services du non-marchand mais ceci en vertu de la nature des services offerts. Dans le même ordre d'idée, les subventions aux premiers travailleurs engagés devraient être remplacées par des subsides à la création d'entreprises.

Comparée à ce qui se pratique actuellement, la durée des subventions temporaires à l'embauche devrait être plus courte, certainement s'il s'agit des subventions aux travailleurs peu qualifiés, comme le sont souvent les chômeurs de longue durée. Pour ces travailleurs, le rythme de croissance de la productivité au cours de la période d'embauche est trop lent pour qu'il puisse compenser la baisse de l'allégement au cours du temps : après la période de subvention, le travailleur sera très souvent licencié. Néanmoins, une subvention temporaire brève permet d'accroître l'efficacité du processus d'appariement entre les employeurs et les demandeurs d'emploi : elle incite les premiers à entamer une relation de travail avec des populations en moyenne moins favorisées et la période temporaire d'embauche permet de révéler de l'information sur les qualités du travailleur.

Selon notre analyse, l'emploi d'une grande proportion de travailleurs faiblement qualifiés est avant tout contraint par le manque de demande de travail (c'est à dire d'offre d'emploi) en raison des niveaux de coûts salariaux. Stimuler l'offre de travail (c'est à dire la demande d'emploi) par de nouvelles mesures d'allégement (para-)fiscal au bas de l'échelle des revenus du travail devrait principalement aggraver le problème du chômage pour ces groupes de population. Par ailleurs, même si la trappe à chômage pose un problème pour certains travailleurs, les étu- 


\section{... Conclusions}

Cibler intelligemment

\section{Modération salariale, assurance-chômage et formation}

des étrangères nous permettent de conclure que le bonus crédit d'impôt n'augmente pas toujours le taux d'emploi et peut même le réduire pour certains travailleurs.

Il est vrai toutefois que des emplois vacants assez peu qualifiés demeurent à tout moment non pourvus. Avant d'incriminer les conditions salariales de tout travail faiblement qualifié, il y a lieu de prendre en compte d'autres facteurs susceptibles d'expliquer ce phénomène. Par exemple, les postes d'emploi non pourvus sont souvent spécifiques au sens où ils sont associés à des conditions de travail précaires : horaires irréguliers ou longs, travail dangereux ou de nuit, etc. En l'absence de compensation monétaire ou non monétaire de ces conditions précaires dans ces emplois spécifiques, il est compréhensible que les travailleurs ne les acceptent pas. Par ailleurs, d'autres éléments peuvent expliquer la présence d'offres d'emploi peu qualifié non satisfaites : information imparfaite sur les offres et les candidats, discriminations à l'embauche, offre inappropriée de crèches et de garderies scolaires, problèmes de mobilité, etc.

Lorsqu'on alloue une diminution de charge, il s'agit de minimiser les effets de substitution et de déplacement. Ces effets sont surtout importants s'il existe des membres de la population active non-bénéficiaires de la subvention ayant une productivité similaire à celle des travailleurs subventionnés. Il faut ainsi surtout éviter de cibler le dispositif via des critères, tels que l'âge, qui ne sont pas fortement liés à la productivité. Le niveau des salaires, la durée de chômage ou le niveau d'étude semblent mieux rencontrer cette exigence. En outre, même pour ces critères, il faut éviter tout clivage brusque (toute séparation tranchée) entre les publics éligible et non éligible (par exemple, en disant que seuls les chômeurs inoccupés depuis plus de 2 ans sont éligibles).

Cet article s'est penché sur la question d'une politique du marché de travail : l'allégement des charges sociales. Vu leur proximité, il nous a paru nécessaire d'aborder également les formules de type crédit d'impôt. La politique d'emploi ne doit bien sûr pas se limiter à cela. Nous avons déjà mentionné qu'une réforme rendant l'assurance chômage plus incitative à la recherche d'emploi est souhaitable. Sous peine de dégrader fortement le bien-être des populations peu qualifiées, elle n'apportera toutefois pas à elle seule une réponse au chômage structurel. Par ailleurs, il faudra sans doute davantage coupler des subventions à des formations (suffisamment longues) en entreprise. A cette fin, un système de certification des formations en entreprise devrait être instauré pour que les compétences apprises puissent être reconnues au-delà de l'entreprise formatrice. Enfin, le maintien de notre compétitivité internationale est essentiel. La modération salariale est ici un des facteurs essentiels (voir le numéro 6 de Regards Economiques). Elle doit aussi être conçue dans la perspective des réformes préconisées plus haut : il est inévitable qu'une part des allégements de cotisations patronales se retrouve sous la forme de salaires nets - et ceci rend le bonus-crédit d'impôt d'autant moins nécessaire. Mais, il importe de maitriser strictement cet effet. Dans ce but, on n'échappera donc pas à une modération salariale des salaires bruts, imposée par une norme ou, mieux, voulue par les partenaires sociaux eux-mêmes.
Bart Cockx, Henri Sneessens, et Bruno Van der Linden sont professeurs au département des sciences économiques de l'UCL et chercheur à l'IRES.

Bruno Van der Linden est également chercheur qualifié du FNRS.
Bart Cockx

Henri Sneessens

Bruno Van der Linden 
Annexe

\section{Présentation des trois évaluations macro-économiques des allégements de cotisations sociales patronales en Belgique}

Les estimations de Sneessens et de Shadman-Mehta (2000) présentent notamment l'originalité d'avoir pour la première fois en Belgique décomposé la main d'œuvre par niveau de qualification. Auparavant, Bréchet et al (1995) avaient exploité une désagrégation sectorielle. Sneessens et Shadman-Mehta se sont naturellement heurtés à la difficulté de réunir des statistiques macroéconomiques par niveau de qualification. Ils évaluent l'effet d'une baisse du taux de cotisations patronales sur le salaire des "peu qualifiés" (nouvelle recrues ou non) s'élevant à 6 points de pourcentage ( $21 \%$ des charges sociales initiales). L'ordre de grandeur global de la baisse de cotisation est voisin de celui de Bréchet et al (1995) et représente globalement une masse budgétaire d'environ $1 \%$ du PIB. Sneessens et Shadman-Mehta confirment que l'effet final sur les salaires et sur l'emploi dépend fortement de la sensibilité des salaires (des "peu qualifiés") au taux de chômage. Dans le cas le plus probable, les autres paramètres de la (para-)fiscalité demeurant inchangés, on peut à terme en attendre une hausse de l'emploi des "peu qualifiés" de 83.000 unités et de l'emploi 'plus qualifié' de 33.000 unités (soit une hausse relative de 6,7 et de 1,4\% respectivement). Ces effets sont basés sur une calibration du modèle relative à l'année 1994 .

Utilisant une nouvelle version du modèle macro-sectoriel HERMES, Stockman (2002) compare trois scénarios d'allégement des cotisations sociales sous deux modes de formation des salaires. Les trois scénarios sont : (1) une baisse des contributions sociales concentrées sur les bas-salaires (moins de $4.685 €$ de salaire brut aux prix de 2000) qui représente ex ante $0.05 \%$ du PIB en 2001 ; (2) une mesure de même ampleur concentrée sur les salaires supérieurs à $4.685 €$; (3) une mesure de même ampleur appliquée à tous les salariés des secteurs privé et sans but lucratif. Soulignons que l'ampleur de la mesure est environ 20 fois moindre que dans les études précédentes. Les salaires bruts ne peuvent dans un cas, par hypothèse, pas bénéficier des baisses de cotisation. Dans l'autre configuration, les salaires bruts sont librement négociés. Le premier mode de formation des salaires apparaît plus favorable à l'emploi avec respectivement, selon le scénario, $4.040,1.530$ et 1.840 personnes supplémentaires en emploi en 2007. L'effet tombe respectivement à $2.380,1.100$ et 610 unités si les salaires sont négociés librement. On constate aussi les effets plus favorables du ciblage sur les bas salaires. Il faut enfin signaler que la réforme ne s'autofinance que de manière partielle.

Pierrard et Sneessens (2003) réexaminent l'efficacité de subventions ciblées sur les bas salaires lorsque le chômage des peu qualifiés est la conséquence à la fois d'un progrès technique biaisé et d'un effet d'éviction. L'évaluation est basée sur un modèle macroéconomique calibré sur l'année 1996. Les salaires des travailleurs qualifiés sont librement négociés ; ceux des moins qualifiés évoluent de façon à garder le salaire net relatif inchangé. Le modèle reproduit assez bien l'évolution observée après 1975 (hausse généralisée du chômage, modérée pour les plus qualifiés, très forte pour les moins qualifiés). Une réduction de charges sociales de $10 \%$ sur les bas salaires (masse globale : environ $1,5 \%$ du PIB) financée par un impôt forfaitaire permet de créer quelque 87.000 emplois supplémentaires (68.000 pour les travailleurs peu qualifiés, 19.000 pour les qualifiés). L'effet de la subvention sur l'emploi des moins qualifiés est atténué par la hausse des salaires nets $(+2 \%)$ et par la concurrence des travailleurs plus qualifiés. Les créations d'emplois sont plus nombreuses $(20 \%$ de plus) lorsque la mesure est financée par une hausse de la taxation des salaires plus élevés, qui freine les revendications salariales. 


\section{Références}

Blanchard O. et J. Wolfers (2000) "The Role of Shocks and Institutions in the Rise of European Unemployment: the Aggregate Evidence”, Economic Journal, 110, 1-33.

Blundell, R. and C. Meghir, 2002, Active Labour Market Policy versus Employment Tax Credits : lessons from recent UK reforms, IFAU discussion paper, 2002 -1.

Bolvig, I., P. Jensen and M. Rosholm, 2003, "The Employment Effects of Active Social Policy", IZA discussion paper, 736, Bonn.

Bréchet, Th., L. Lemiale, C. Streel et P. Van Brusselen, 1995, Les effets d'une politique de réduction du coût salriale ciblée sur les bas salaires, Cahiers économiques de Bruxelles, 146, pp. 161-185.

Bresnahan, T.F., E. Brynjolfsson et L.M. Hitt , (2002), Information Technology, Workplace Organization, and the Demand for Skilled Labor: Firm-level Evidence, Quarterly Journal of Economics, 117(1), p.339-76.

Burggraeve K. and Ph. Du Caju, 2003, The case of reductions of employers' social security contributions under a wage norm regime with automatic indexing of wages, Banque Nationale de Belgique, Bruxelles, working paper 36.Cahuc P. et A. Zylberberg, 2003, Microéconomie du marché du travail, La Découverte, Paris, Collection Repères, 354.

Cahuc, P., 2002, Baisser les charges sociales: jusqu'où et comment? , Centre d'Observation d'Economie, Chambre de Commerce et d'Industrie de Paris, Document , $\mathrm{n}^{\circ}$ 59. Paru également dans la Revue française d'Economie, 17, janvier 2003.

Cahuc P. et A. Zylberberg, 2001, Le marché du travail, De Boeck Université, Bruxelles, Collection Balises.

Cahuc, P., 2001, La Prime pour l'Emploi à la lumière des expériences étrangères, Centre d'Observation d'Economie, Chambre de Commerce et d'Industrie de Paris, Document $\mathrm{n}^{\circ} 56$.

Calmfors, L., A. Forslund et M. Hemström, 2002, Does active labour market policy work? Lessons from the Swedish experiences , IFAU Office of Labour Market Policy Evaluation, Working Paper 2002-4.

Caroli, E., et J. Van Reenen (2001), Skill-biased Organizational Change? Evidence from a Panel of British and French Establishments, Quarterly Journal of Economics, 116(4), p.1449-92.

Cockx B., C. Göbel, S. Robin et B. Van der Linden, 2004, Politiques d'activation pour des jeunes chômeurs de longue durée. Une évaluation, rapport final d'une étude commandité par la Politique scientifique fédérale, à paraître.

Cockx, B. et J. Ries, 2003, Impact sur la réinsertion de la fin de droit à l'indemnisation dans l'assurance chômage, Partie 2 dans Cockx, B., L. De Lathouwer, C. Bogaerts, J. Ries et $\mathrm{K}$. Van den Bosch, L'impact des sanctions relatives aux allocations de chômage sur la réinsertion et sur la pauvreté, rapport final d'une étude commandité par la Politique scientifique fédérale, à paraître.

Cockx B. and M. Dejemeppe, 2002, Duration dependence in the exit rate out of unemployment in Belgium: Is it true or sprurious? IZA, Institute for the Study of Labor, 632, Bonn, à paraître dans le Journal of Applied Econometrics.

Crépon B. et R. Desplatz, 2001, Une nouvelle évaluation des effets des allégements de charges sociales sur les bas salaires, Economie et Statistique, 348, pp. 3-24.

D'Addio, A., I. De Greef and M. Rosholm, 2002 , An econometric analysis of unemployment trap for Belgium, IZA, Institute for the Study of Labor, 669, Bonn. Version révisée.

Dejemeppe M., 2003, A Complete Decomposition of Unemployment Dynamics Using Longitudinal Grouped Duration Data, Institut de Recherches Economiques et Sociales, Université Catholique de Louvain, mimeo.

Dor E., B. Van der Linden and M. Lopez-Novella, 1997, On labour market policies and aggregate unemployment outflows, Oxford Bulletin of Economics and Statistics, vol 59, 1, pp. 109-132.

Gerfin, M., M. Lechner and H. Steiger, 2002, Does Subsidised Temporary Employment Get the Unemployed Back to Work ? An Econometric Analysis of Two Different Schemes, IZA Discussion paper 606, Bonn.

Heckman J., R. Lalonde and J. Smith, 1999, The Economics and Econometrics of Active Labor Market Programs , in O. Ashenfelter et D. Card (eds.), Handbook of Labor Economics, vol. III, Amsterdam : North Holland. 


\section{... Références}

\section{Regards Économiques IRES-UCL}

Place Montesquieu, 3

B1348 Louvain-la-Neuve

site Web: http://regards.ires.ucl.ac.be mail: regards@ires.ucl.ac.be tél. 010/47 4146 ou 010/47 3426

Directeur de la publication :

Vincent Bodart

Rédactrice en chef :

Muriel Dejemeppe

Comité de rédaction :

Paul Belleflamme, Vincent Bodart,

Raouf Boucekkine, Isabelle Cassiers,

Muriel Dejemeppe, Jean Hindriks,

Vincent Vandenberghe,

Vincent Vannetelbosch

Secrétariat : Anne Davister,

Yolande de Ryckel

Graphiste : Dominos
Hendrickx K., Ch. Joyeux, M. Lopez-Novella, L. Masure et P. Stockman, 2003, un nouveau modèle macro-économétrique du marché du travail: estimation, simulation de base et simulation de politiques d'emploi, Working Paper 13-03 du Bureau fédéral du Plan, Bruxelles, avril.

Joyeux Ch. en P. Stockman, 2002, Patronale bijdrageverminderingen en loonkostvoet: een partiële analyse voor 1995-2000, Bureau fédéral du Plan, Bruxelles, Rapport au Conseil Central de l'Economie.

Lalive, R., J. Van Ours and J. Zweimüller, 2002, The Impact of Active Labor Market Programs on the Duration of Unemployment, mimeo, IEW, University of Zürich.

Lopez-Novella, M. (2003) Effets de certains subsides temporaires à l'embauche: une analyse micro-économique des plans plus et du plan avantage à l'embauche, Working paper 16-03 du Bureau fédéral du Plan, Bruxelles.

Machin S. and A. Manning, 1999, The causes and consequences of long-term unemployment in Europe in Ashenfelter O. and D. Card, Handbook of labor economics, NorthHolland, vol. 3C, pp .3085-3139.

Martin, J. P. and D. Grubb, 2001, What works and for whom: a review of OECD countries' experiences with active labour market policies , IFAU Office of Labour Market Policy Evaluation, Working Paper 2001-14.

OCDE, 2003, Etude économique de l'OCDE, Belgique, Février, OCDE, Paris.

Ooghe E., E. Schokkaert and Flechet J., 2000, The incidence of social security contributions: An empirical analysis, Discussion paper DPS 00.23, Center for Economic Studies, Department Economie, KUL.

Orszag, J.M. and D. Snower , 2003, Designing employment subsidies, Labour Economics, 10(5), pp. 557-572.

Pierrard O. et H. Sneessens (2003a) Low-Skilled Unemployment, Biased Technological Shocks and Job Competition, IZA Discussion Paper No. 784, Institute for the Study of Labor, Bonn.

Pierrard O. et H. Sneessens (2003b) Inadéquations, déqualifications et chômage, mimeo, IRES, Département des sciences économiques, Université catholique de Louvain.

Sneessens H. et F. Shadman, 2000, Analyse macro-économique des effets des réductions ciblées des charges sociales, Revue Belge de Sécurité Sociale, 42, Septembre, pp.613-630.

Stockman P., 2002, General and selective reductions in employer social-security contributions in the 2002 vintage of HERMES - A revision of WP 8-01, Federal Planning Bureau, Brussels, working paper 6-02.

Van der Linden B., 2003, Unemployment insurance and training in an equilibrium matching model with heterogeneous agents, Discussion Paper de l'IRES, 2003-01, Université Catholique de Louvain,.

Van der Linden B. et E. Dor, 2002, Effets macroéconomiques de l'indemnisation et de la formation des chômeurs en Belgique?, 15ième Congres des Economistes belges de Langue française, Commission 3 : Politiques actives pour l'emploi, 28-29 novembre 2002.

Van der Linden, B., 1997, Effets des formations professionnelles et des aides à l'embauche : exploitationd'une enquête auprès d'employeurs, Economie et Prévision, 130, pp.113-30.

Van Poeck A., P. Van Rompuy, J. Vanneste, G. De Bruyne, J. Crappe, Ph. De Caju, G. Groffen, P. Diaz en I. Lathouders, 1996, Fiscaliteit, parafiscaliteit en de werking van de arbeidsmarkt in België, Programma Publieke Economie, Fiscaliteit, Federal Diensten voor Wetenschappelijke, Technische en culturele aangelegenheden, Brussel.

Van Poeck A., J. Vanneste, en Ph. De Caju P, 1998, Leidt een vermindering van de bijdragen van Sociale Zekerheid tot meer werkgelegenheid in België?, Cahiers économiques de Bruxelles, 157, 1er trimestre, pp. 35-63. 